\title{
Performance evaluation of NB-IoT in-band deployment mode in suburban area
}

\author{
Karina Turzhanova1, Sergey Konshin ${ }^{2}$, Valery Tikhvinskiy ${ }^{3}$, Alexandr Solochshenko ${ }^{4}$ \\ ${ }_{1,2,4}$ Almaty University of Power Engineering and Telecommunications named after Gumarbek Daukeev, Almaty, Kazakhstan \\ ${ }^{3}$ FSUE Radio Research and Development Institute, Moscow, Russian Federation
}

\begin{abstract}
Article Info
Article history:

Received Feb 28, 2021

Revised Jun 13, 2021

Accepted Jun 25, 2021

\section{Keywords:}

In-band mode

LTE

NB-IoT

Network performance

QoS

ABSTRACT

There given to discuss the study results about one of three deployment scenarios performance (in-band deployment mode) of the narrow-band internet of things (NB-IoT) technology. The study is carrying out with help of simulation modeling and experimental testing of the main network parameters, namely: radio coverage, network capacity, user experience, and their dependencies on each other. Comparison of the results of a physical experiment and simulation modeling shows their high convergence and confirms the adequacy of the applied testing methodology. As a case scenario provided an example of NB-IoT implementation on a $4 \mathrm{G}$ mobile network in the $800 \mathrm{MHz}$ band, in a suburban area for remote metering applications. The article presents the applying testing methodology of NB-IoT that adapted to the local conditions of radio network planning. Based on the obtained data, adducing the main conclusions about the feasibility of using an in-band scenario for deploying NB-IoT on a $4 \mathrm{G}$ network in a suburban environment.
\end{abstract}

This is an open access article under the CC BY-SA license.

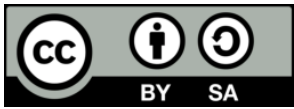

\section{Corresponding Author:}

Karina Turzhanova

Department of Telecommunication Networks and Systems

Almaty University of Power Engineering and Telecommunications named after Gumarbek Daukeev

126/1 Baytursynuli Road, Almaty 050013/A15G8M2, Kazakhstan

Email: karinaturzhanova@gmail.com

\section{INTRODUCTION}

The third generation partnership project (3GPP) in release 13 [1] presented the first version of the specification of standard for cellular communication for subscriber devices and sensors, designed to transfer small amounts of data over long distances. Due to the ability to transmit data in a narrow frequency band, using only 180 $\mathrm{kHz}$ of operation bandwidth, this standard called narrow band internet of things (NB-IoT). NB-IoT technology mainly inherited from LTE, but has many special features and benefits, including: i) improved coverage, ii) massive connections, iii) reduced complexity and cost of user equipment (UE), iv) long battery lifetime due to low power consumption, v) reliability and backward compatibility [2], [3]-[5]. Additional benefits of NB-IoT technology become deployment flexibility [6] and high spectral efficiency achieved using three NB-IoT deployment scenarios: i) standalone, ii) in-band and iii) guard-band proposed in 3GPP specification TR 45.820 [7].

Standalone mode based on replacing one or more GSM carriers with $200 \mathrm{kHz}$ bandwidth with NBIoT carriers with $180 \mathrm{kHz}$ bandwidth. In this case, every $10 \mathrm{kHz}$ acts as a guard interval on either side. The standalone mode presented in Figure 1(a). In-band mode, shown in Figure 1(b), allows allocating physical resource blocks (PRB) within the long term evolution (LTE) spectrum for NB-IoT technology. Finally, guardband mode allows implementing NB-IoT technology by reusing of guardbands of the LTE channel, thereby eliminating the necessity of using new spectrum [8]-[9]. In Figure 1(c) depicts this scenario. 


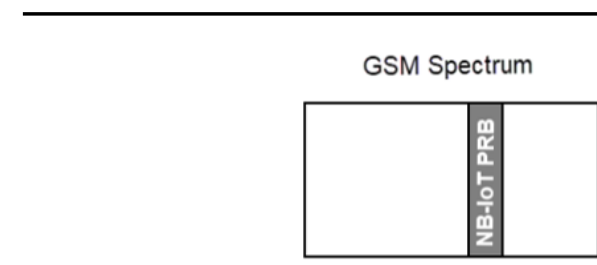

(a)

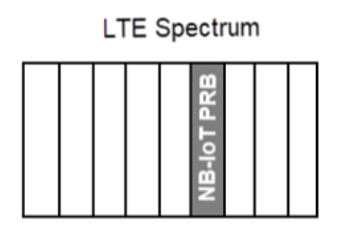

(b)

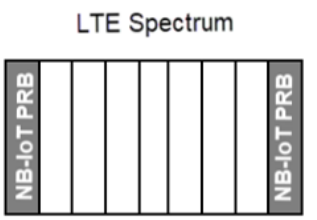

(c)

Figure 1. NB-IoT deployment scenario, adopted from [10]; (a) standalone allocation (180 kHz); (b) in-band allocation $(180 \mathrm{kHz})$; (c) guard-band allocation $(180 \mathrm{kHz})$

NB-IoT technology using the principle and architecture of existing cellular networks, which makes it possible to simplify and speed up the deployment of this technology [11], and almost completely reuse the existing equipment of LTE base transmitting stations (BTS) [12]. According to global system for mobile communications association (GSMA) data [13] as of December 2020, more than 105 NB-IoT networks were launched everywhere, most of which are implemented mainly in guard-band and standalone modes. This associated with the necessity to replace LTE PRB by NB-IoT, the in-band deployment mode requires more attention in radio-frequency planning and NB-IoT deployment [14], has some limitations on the output power of equipment and a large probability of interference occurrence. All of this can degrade the network performance.

For example, in paper [15], compared three NB-IoT deployment scenarios in terms of coverage, network capacity, occupied bandwidth, spectrum and delay. The authors note that in-band deployment mode provides the worst performance in terms of radio coverage and capacity, but at the same time, the spectral efficiency of this scenario is much higher. Unfortunately, the presented information is compressed and the exponents nominal values not given in the work.

Persia and Rea [16] went further and carried out a theoretical analysis of the NB-IoT capacity for advanced measurement applications depending on the building density-urban and suburban areas. The results of empirical studies of deployment scenarios are given in papers [17]-[19]. Nashiruddin et al. [18] describe step by step the used methodology of planning and testing NB-IoT network coverage in the $939.8 \mathrm{MHz}$ frequency range. Here, due to better coverage, standalone mode chosen as the recommended deployment scenario for NBIoT in Indonesian urban area. In work [17] the authors continue more detailed experimental studies of the NBIoT networks performance for in-band and standalone modes. In the conditions of the specified geographical area, the authors also concluded that standalone mode is the best scenario and, with a value of up to -126.30 $\mathrm{dBm}$ provides more stable NB-IoT connection. The study limited in determining the area of terrain coverage.

On the other side, particular the in-band deployment mode makes NB-IoT a standout among all other LPWA technologies [20]. Since for operators with predominantly available LTE spectrum, this scenario allows the most spectrum-and cost-efficient implementation of NB-IoT. As the main performance parameters, the authors of work [19], [21]-[23] most often use maximum coupling loss (MCL), uplink and downlink throughput, exception report latency, co-channel and adjacent channel interference, network coverage and network capacity.

Listed above studies have made an important contribution to the study of the deployment of NB-IoT technology specifics. However, neither one of the works provides a detailed analysis of the in-band mode and its performance (main parameters) in the case of implementation on a real cellular LTE network. Despite the indisputable necessity of the above theoretical studies (for example, to understand the theoretical upper and lower values of the parameters), we consider that particularly empirical approach provides the most complete understanding of the functioning of the real-world NB-IoT network and its behavior in the case of a real load on the network. This study is of practical significance for mobile network operator (MNO) and is an addition to previous related works.

In this paper, we present for discussion the results of simulation modeling and an empirical study of the performance of an in-band NB-IoT technology deployment scenario launched on a 4G network in the 800 $\mathrm{MHz}$ band in the suburban area. Research is being conducted to obtain real values of the key performance exponents of NB-IoT within launching in a test cluster of a $4 \mathrm{G}$ network and to show the benefits of this deployment scenario. Such studies are valuable because they allow us to get reliable information about user and network behavior in advance and take measures for improving network performance before putting it into commercial operation [17]. The main contribution of the research are as follows:

- $\quad$ The general methodology for testing 4G networks has been adapted to the requirements of the in-band scenario for deploying NB-IoT technology;

- $\quad$ Presented for discussion the results of simulation modeling and experimental research of network performance within using an in-band scenario for deploying NB-IoT technology; 
- The main conclusions and recommendations for MNO are given in the case of using the in-band scenario of deploying NB-IoT technology on 4G networks.

The remaining part of the article structured as follows. In the section 2 describes the testing methodology used. The test results presented in section 3, and in section 4 presented brief conclusions.

\section{RESEARCH METHOD}

\subsection{Methodology}

The main objective of this work involves in study the performance of the in-band scenario of NBIoT, implemented on a test section of a $4 \mathrm{G}$ network in the $800 \mathrm{MHz}$ frequency range in the suburban area. For this carrying out the simulation modeling in the specialized software environment Forsk Atoll 3.3.2 and provides an experimental study. The research methodology consists of three fundamental tasks:

- $\quad$ Modeling the performance of NB-IoT networks in the in-band mode using the Forsk Atoll 3.3.2 software environment;

- $\quad$ Experimental study of NB-IoT network performance in the in-band mode on a test cluster of a $4 \mathrm{G}$ network, consisting of 6 BTS;

- $\quad$ Collection and analysis of the main parameters of network performance in the provision of NB-IoT services.

\subsection{Network performance indicators}

The following key performance indicators (KPI) selected as the main parameters of network performance in the provision of NB-IoT services:

\subsubsection{Radio coverage}

Assesses the coverage area of IoT services. Defined by the Reference Signal Received Power (RSRP) parameter and measured in $\mathrm{dBm}$. NB-IoT RSRP reporting range is $-44-140 \mathrm{dBm}$. The RSRP value is lower the further the UE is from the base station, and vice versa [24]. Thus, the distance of the UE from the BTS directly affects the RSRP.

\subsubsection{Network quality}

Determines information transmission quality, namely the level of the useful signal, subject to the influence of third-party noise and interference. Described by the signal to interference + noise ratio (SINR) parameter and measured in $\mathrm{dB}$. SINR using as the main parameter of the network quality, since it reported by the subscriber terminal and most accurately describes the radio conditions. NB-IoT SINR reporting range is $-20-20 \mathrm{~dB}$. The higher the SINR value, the better signal and network quality in general. With a negative SINR value, the noise level exceeds the desired signal level. For SINR, there is no direct dependence on the distance between UE and BTS. SINR and RSRP parameters are one of the main KPIs designed to measure the radio conditions of $4 \mathrm{G}$ networks [25].

\subsubsection{User experience}

Determines the quality of the provided communication services from the client's view point and consists of such parameters as uplink/downlink (UL/DL) throughput, cell edge throughput, data traffic, and call drop rate. Since the point at issue is technology, which designed to transfer small data amounts that are not critical to breaking of connections, we will restrict ourselves to measuring UL/DL throughput.

\subsection{Test environment}

At present times, the most prospective area of NB-IoT application is remote metering applications, where NB-IoT technology allow to data collection and remote control for suppliers of electricity, heat energy and water utilities [26]-[27]. This user case taken as the basis for modeling [28] and testing the performance of an NB-IoT network when using in-band deployment mode as shown in Figure 2. Simulation modeling aimed at reproducing the conditions of operation of the NB-IoT on the LTE network that are closest to real ones. To achieve that we use the Forsk Atoll 3.3.2 software, the signal propagation model-Cost231 Hata model in suburban environment [29]:

$$
\begin{aligned}
& P L_{\text {suburban }}=46.3+33.9 \log _{10}(f)-13.82 \log _{10}\left(h_{b}\right)- \\
& a h_{m}+\left(44.9-6.55 \log _{10}\left(h_{b}\right)\right) \log _{10}(d)+C_{m},
\end{aligned}
$$

where $P L_{\text {suburban }}$ is a propagation loss in Suburban area, $f$ is the transmission frequency, $h_{m}$ is the height of the mobile device in $\mathrm{m}, h_{b}$ is the height of the BTS in $\mathrm{m}, d$ is the distance between the receiver and the transmitter in $\mathrm{km} \mathrm{[30],} C_{m}$ is the correction factor defined as 0dB for suburban area. 
In Forsk Atoll 3.3.2, in the simulation settings, we indicate the existing environmental conditions on the test cluster of the $4 \mathrm{G}$ network, namely, a suburban cluster zone with 6 BTS Figure 3 . The coverage radius of the base station is approximately $3 \mathrm{~km}$. User equipment randomly distributed in the selected cluster. The settings in the simulator setting up in accordance with the local radio network planning conditions and the existing settings of a test $4 \mathrm{G}$ network. The input data for the modeling is presented in Table 1.

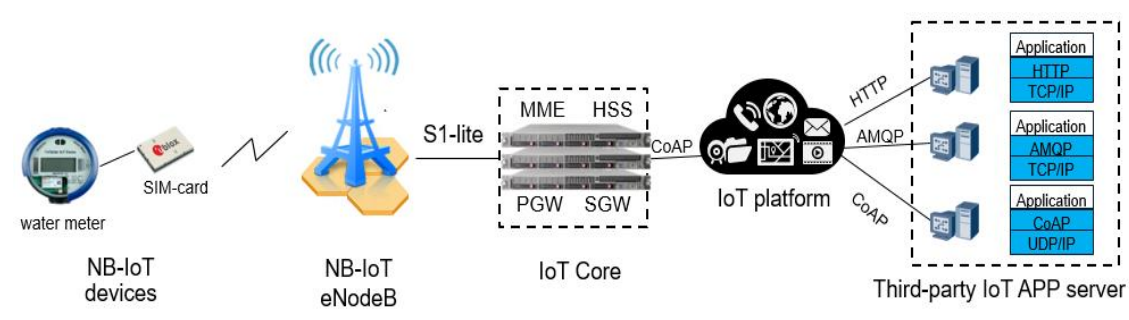

Figure 2. Network architecture of NB-IoT network for water meter application

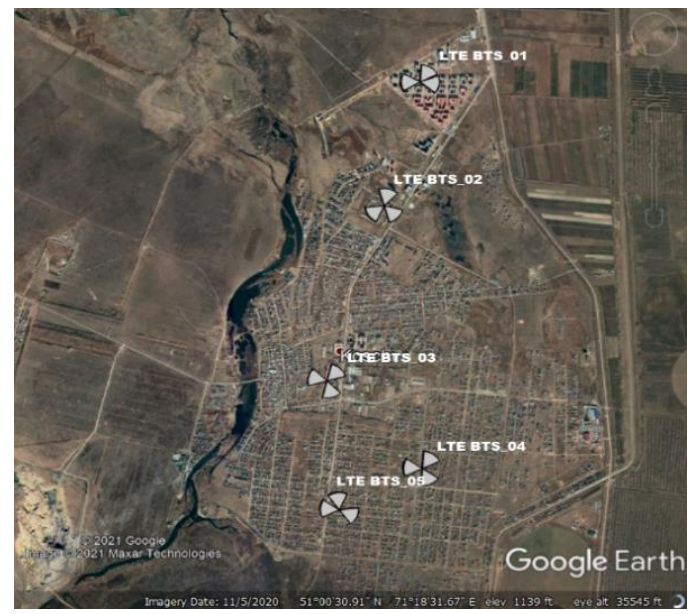

Figure 3. Site location and cell-site orientation in Google map

Table 1. Test assumptions

\begin{tabular}{ll}
\hline \multicolumn{1}{c}{ Parameter } & \multicolumn{1}{c}{ Variable } \\
\hline Working mode & In-band \\
Architype & Suburban \\
Population density & $1636 / \mathrm{km}^{2}$ \\
NB-IoT (UE) device density & $800 / \mathrm{km}^{2}$ \\
Scenario & $15 \mathrm{sectors}(5$ sites) operating in $800 \mathrm{MHz}$ \\
Inter-site distance (ISD) & $1.0-2.8 \mathrm{~km}$ \\
Base-station antenna height & $18-30 \mathrm{~m}$ \\
Operating bandwidth & $10 \mathrm{MHz}$ (LTE), 180kHz (NB-IoT) \\
BTS transmit power for NB-IoT & $35 \mathrm{dBm}$ \\
Antenna configuration & BTS: 1T 2R; UE: 1T 1R \\
LTE BTS sector load & $<80 \%$ \\
Available resources & $1 \mathrm{PRB} /$ device \\
BTS receiver noise figure & $3 \mathrm{~dB}$ \\
\hline
\end{tabular}

\section{RESULTS AND DISCUSSION}

The section shows the results of a network performance study for an in-band NB-IoT deployment scenario in a $4 \mathrm{G}$ test cluster in a suburban area. The analysis carried out based on measuring the previously presented parameters of RSRP, SINR, UL and DL user throughput, as well as their dependencies on each other according to the developed testing methodology. These metrics are most suitable for describing the performance of NB-IoT technology [21]. 


\subsection{Radio coverage with network quality}

An analysis of parameters of coverage and quality of the NB-IoT network in suburban area shown in Figures 4 and 5. Research results show that:

- Loss of communication between the base transceiver station and the user equipment occurs when the RSRP threshold value reached of $-130 \mathrm{dBm}$ Figure 4. At the same time, only $2.2 \%$ of computation zones have a coverage area with a signal of $-130 \mathrm{dBm}$ and below Figure 5, which is due to the distance from the base transceiver station and the conditions of the geographic area.

- The average of the best signal level for the suburban area is $-92.48 \mathrm{dBm}$. At a distance of $2.5-2.7 \mathrm{~km}$ from the base station, the signal level (RSRP) drops to $-120 \mathrm{dBm}$. Provided that the receiver sensitivity is at least $-141 \mathrm{dBm}$, the user terminals still can connect to the base station and transmit data on this distance.

- Under conditions of the local terrain, the average zone edge of base station coverage is $1.37 \mathrm{~km}$, which corresponds to an RSRP value of $-98.3 \mathrm{dBm}$. In this case, the optimal SINR value is $1.08 \mathrm{~dB}$ Figure 5 .

It should be noted that the results of the RSRP and SINR study are not absolute, as they depend on many factors, such as: the loading and operating conditions of the base station, the sensitive of UE's receiving antenna and weather conditions.

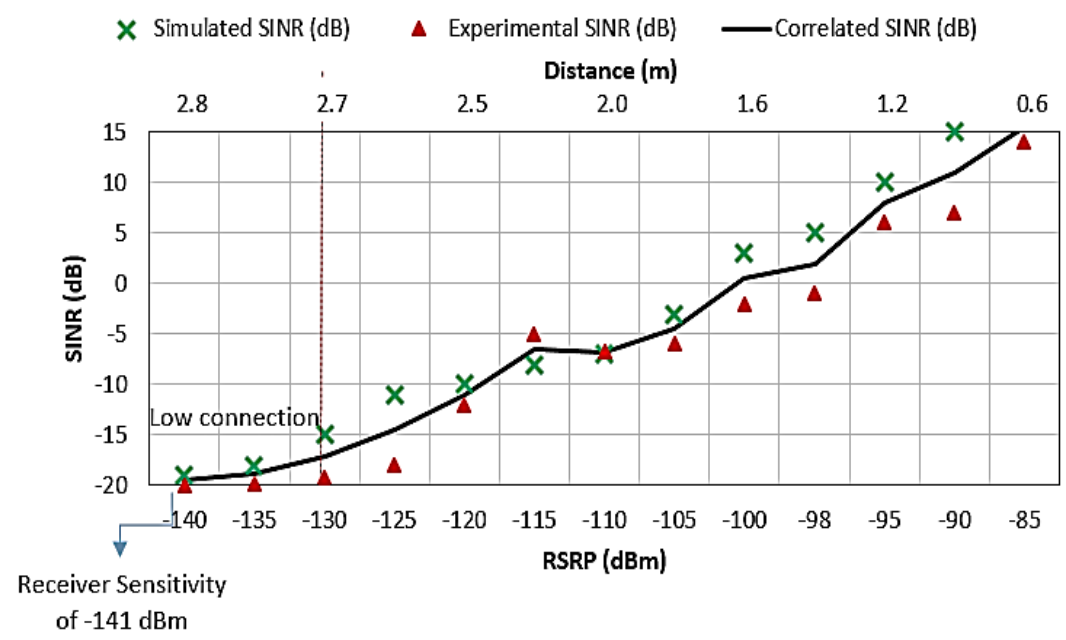

Figure 4. The dependence of network quality (SINR) from radio coverage (RSRP)
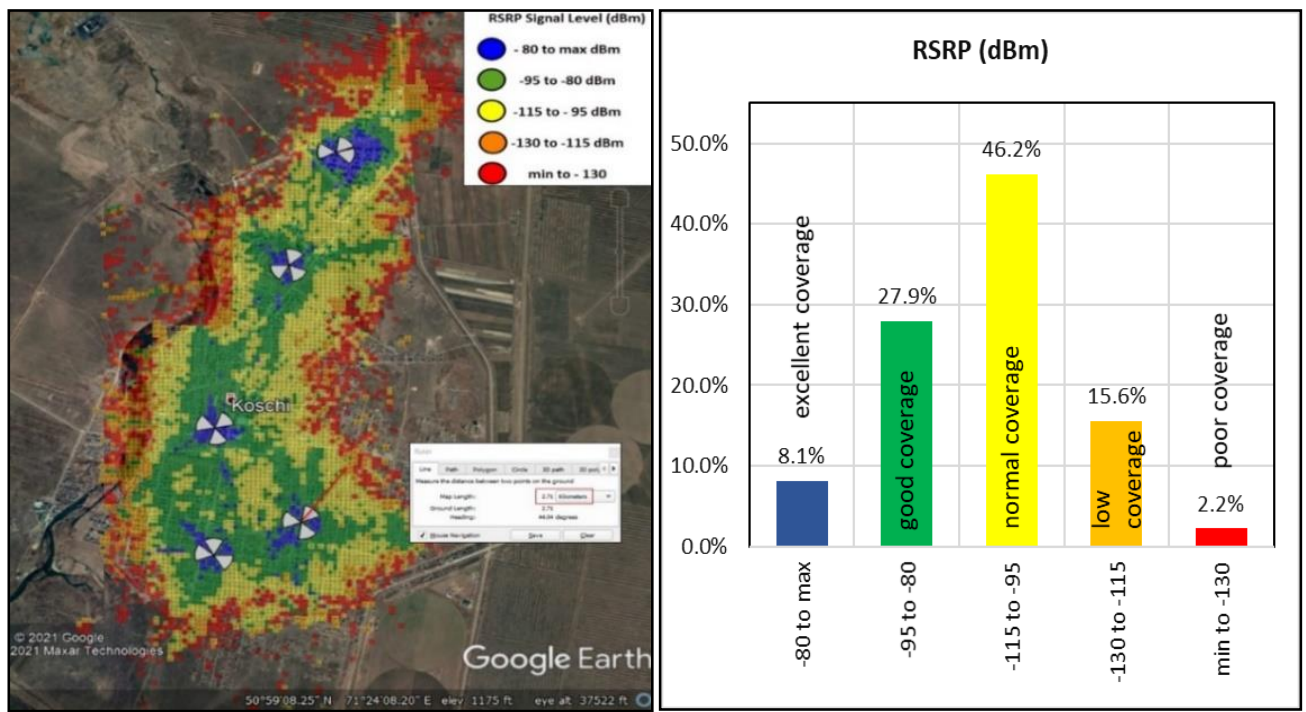

Figure 5. Coverage prediction and histogram of signal level (RSRP) for Suburban scenario 


\subsection{Uplink and downlink throughput with radio coverage}

The next evaluation was conducted to measure UL and DL throughput provided by NB-IoT in dependence on signal strength (RSRP). For this were sent one hundred packets per 512 bytes. All packets successfully received at the receiving side. Figure 6 illustrates the DL throughput for different signal levels. The DL throughput remained nearly constant $(20.2 \mathrm{kbps})$ until about $-115 \mathrm{dBm}$ of RSRP $(2.1 \mathrm{~km})$, which allow installing NB-IoT UE in a wide variety of suburban locations.

According to papers [7], [31], the in-band NB-IoT deployment mode has peak UL and DL throughput -22.7 and $62.5 \mathrm{kbps}$, respectively. At the same time, the research results presented in Table 2 show that the maximum possible speed obtained in modeling and experimental research is not much different from the theoretical one. At the same time, the deviation between the model and experimental speed values does not exceed $20 \%$. The reason for this can be both external radio conditions and restrictions on the side of user equipments, as well as simulation settings in the software environment.

X Simulated DL Throughput (kbps) $\Delta$ Experimental DL Throughput (kbps) — Correlated DL Throughput (kbps)

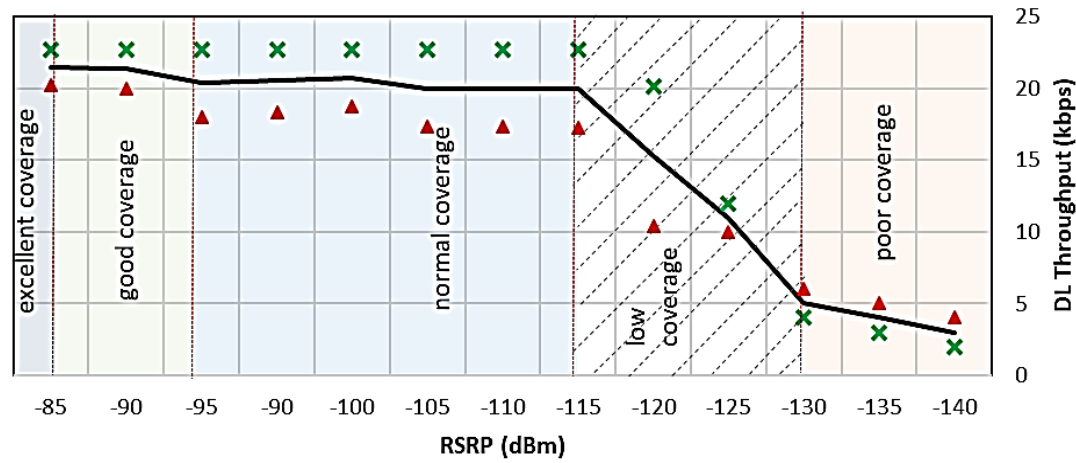

Figure 6. DL Throughput for different signal levels (RSRP)

Table 2. Comparison of NB-IoT throughput in an in-band deployment

\begin{tabular}{ccccc}
\hline Parameter & $\begin{array}{c}\text { 3GPP Specification } \\
{[7]}\end{array}$ & $\begin{array}{c}\text { Simulated } \\
\text { Value }\end{array}$ & $\begin{array}{c}\text { Experimental } \\
\text { Value }\end{array}$ & $\begin{array}{c}\text { Deviation between model and experimental } \\
\text { value }\end{array}$ \\
\hline $\begin{array}{c}\text { UL throughput, } \\
\text { kbps } \\
\text { DL throughput, } \\
\text { kbps }\end{array}$ & 62.5 & 60.5 & 48.3 & $20 \%$ \\
\hline
\end{tabular}

\section{CONCLUSION}

The in-band deployment scenario allows running NB-IoT technology inside existing 4G networks, which have large coverage and provide the necessary quality of service for the internet of things by today. This is the optimum scenario for connecting of IoT, since it allows telecom operators to use modern equipment without significant hardware changes. On the other hand, in this mode NB-IoT takes away the functional resources of the $4 \mathrm{G}$ cellular network and can create noises (interference), thus reduce the performance of NB-IoT technology and $4 \mathrm{G}$ networks. To make a decision on the usage of in-band scenario for deploying NB-IoT on a $4 \mathrm{G}$ network in a suburban area and for conducting a qualitative evaluation of such network performance, the authors recommend using the proposed method for testing NB-IoT networks and build on the results of the simulation modelling and on experimental network testing. The research results show that the in-band scenario meets the stringent requirements to coverage $(>-130 \mathrm{dBm})$ and network quality $(>-17 \mathrm{~dB})$ on the distance up to $2.7 \mathrm{~km}$. Comparison of network performance parameters that obtained on based on the results of a physical experiment and simulation modeling showed high convergence. The exception was measurements of the DL user experience parameter with a network coverage value of $-95 \ldots-120 \mathrm{~dB}$. Stable transmission rate up to $20.2 \mathrm{kbps}$ in downlink provided on the distance up to $2.1 \mathrm{~km}$. In addition, observed that NB-IoT also provides good coexistence performance with the existing 4G network as during testing significant interference was not observed. Thus, the in-band scenario meets the basic requirements of the 3GPP specification and is optimal for using on a $4 \mathrm{G}$ network in the $800 \mathrm{MHz}$ band in the suburban area to provide remote metering application services. 


\section{REFERENCES}

[1] 3GPP TSG, RAN Meeting №69, "Narrow band IoT (NB-IoT)," RP - 151621, Sep 2015.

[2] Y.-P.E. Wang, et al., "A Primer on 3GPP Narrowband Internet of Things," in IEEE Communications Magazine, vol. 55, no. 3, pp. 117-123, Mar 2017, doi: 10.1109/MCOM.2017.1600510CM.

[3] Y. Sun, F. Tong, Z. Zhang, and S. He, "Throughput Modeling and Analysis of Random Access in Narrowband Internet of Things," in IEEE Internet of Things Journal, vol. 5, no. 3, pp. 1485-1493, Jun 2018, doi: 10.1109/JIOT.2017.2782318.

[4] Y. D. Beyene, et al., "NB-IoT Technology Overview and Experience from Cloud-RAN Implementation," in IEEE Wireless Communications, vol. 24, no. 3, pp. 26-32, Jun 2017, doi: 10.1109/MWC.2017.1600418.

[5] A. D. Zayas and P. Merino, "The 3GPP NB-IoT system architecture for the Internet of Things," 2017 IEEE International Conference on Communications Workshops (ICC Workshops), 2017, pp. 277-282, doi: 10.1109/ICCW.2017.7962670

[6] S. Kiatikitikul and A. Monsakul, "A Performance Study in NB-IoT networks through the IoT Platforms in Thailand," RSU International Research Conference 2019, 2019, pp. 408-415.

[7] 3GPP TR 45. 820, "Cellular system support for ultra low complexity and low throughput internet of things," Release 13, Nov 2015.

[8] R. Ratasuk, N. Mangalvedhe, Y. Zhang, M. Robert, and J. P. Koskinen, "Overview of narrowband IoT in LTE Rel13," 2016 IEEE Conference on Standards for Communications and Networking (CSCN), 2016, pp. 1-7, doi: 10.1109/CSCN.2016.7785170

[9] J. Schlienz and D. Raddino, "Narrowband internet of things whitepaper," White Paper, Rohde\&Schwarz, pp. 1-42, 2016.

[10] K. F. Muteba, K. Djouani, and T. O. Olwal, "Opportunistic Resource Allocation for Narrowband Internet of Things: A Literature Review," 2020 International Conference on Electrical, Communication, and Computer Engineering (ICECCE), Istanbul, Turkey, 2020, pp. 1-6, doi: 10.1109/ICECCE49384.2020.9179427.

[11] M. A. Imran, S. Hussain, and Q. H. Abbasi, "Narrowband Internet of Things (NB-IoT) for Industrial Automation," in Wireless Automation as an Enabler for the Next Industrial Revolution, pp. 65-87, 2020, doi: 10.1002/9781119552635.ch4.

[12] S. K. Routray and S. Mohanty, Principles and Applications of Narrowband Internet of Things (NBIoT), Pennsylvania, USA: IGI Global, 2021.

[13] Mobile IoT Network Launches, 2020. [Online]. Available: https://www.gsma.com/iot/mobile-iot-commerciallaunches/

[14] N. Mangalvedhe, R. Ratasuk, and A. Ghosh, "NB-IoT deployment study for low power wide area cellular IoT," 2016 IEEE 27th Annual International Symposium on Personal, Indoor, and Mobile Radio Communications (PIMRC), 2016, pp. 1-6, doi: 10.1109/PIMRC.2016.7794567,

[15] D. Xue, H. Xu, and P. Li, "An Indoor 3D Positioning Technology Based on NB-IoT," Workshops of the International Conference on Advanced Information Networking and Applications, 2019, pp. 35-43.

[16] S. Persia and L. Rea, "Next generation M2M Cellular Networks: LTE-MTC and NB-IoT capacity analysis for Smart Grids applications," 2016 AEIT International Annual Conference (AEIT), 2016, pp. 1-6, doi: 10.23919/AEIT.2016.7892789.

[17] E. S. Santoso, A. Hidayati, M. Suryanegara, and I. M. Nashiruddin., "NB-IoT Network Planning for Smart Metering Services in Jakarta, Depok, Tangerang, and Bekasi," 2019 16th International Conference on Quality in Research (QIR): International Symposium on Electrical and Computer Engineering, 2019, pp. 1-6, doi: 10.1109/QIR.2019.8898262

[18] M. I. Nashiruddin and A.A.F. Purnama, "NB-IoT Network Planning for Advanced Metering Infrastructure in Surabaya, Sidoarjo, and Gresik," 2020 8th International Conference on Information and Communication Technology (ICoICT), 2020, pp. 1-6, doi: 10.1109/ICoICT49345.2020.9166315.

[19] H. Malik, M. Mahtab Alam, Y. Le Moullec, and A. Kuusik, "NarrowBand-IoT Performance Analysis for Healthcare Applications," Procedia Computer Science, 2019, vol. 130, pp. 1077-1083, doi: 10.1016/j.procs.2018.04.156.

[20] S. Landstrom, J. Bergstrom, E. D. Westerberg, and Hammarwall, "NB-IoT: A sustainable technology for connecting billions of devices," Ericsson Technol. Rev., vol. 4, pp. 2-11, Apr 2016.

[21] A.P. Matz, J.-A. Fernandez-Prieto, J. Cañada-Bago, and U. Birkel, "A Systematic Analysis of Narrowband IoT Quality of Service," Sensors 2020, vol. 20, no. 6, pp. 1-26, 2020, doi: 10.3390/s20061636.

[22] S. Basu, A. Sultania, J. Famaey, and J. Hoebeke, "Experimental Performance Evaluation of NB-IoT," 2019 International Conference on Wireless and Mobile Computing, Networking and Communications (WiMob), 2019, pp. 1-6, doi: 10.1109/WiMOB.2019.8923221.

[23] A. Hoglund, et al., "Overview of 3GPP Release 14 Enhanced NB-IoT," IEEE Network, vol. 31, no. 6, pp. 16-22, 2017, doi: 10.1109/MNET.2017.1700082.

[24] S. Pramono, L. Alvionita, M. Danang, and M. E. Sulistyo, "Optimization of 4G LTE (Long Term Evolution) Network Coverage Area in Sub Urban," in AIP Conference Proceedings, 2020, pp. 1-9.

[25] F. Afroz, R. Subramanian, R. Heidary, K. Sandrasegaran, and S. Ahmed., "SINR, RSRP, RSSI and RSRQ Measurements in Long Term Evolution Networks," International Journal of Wireless \& Mobile Networks (IJWMN), vol. 7, no. 4, pp. 113-123, Aug 2015, doi: 10.5121/ijwmn.2015.7409.

[26] O. Vermesan and P. Friess, "Internet of Things Applications: From Research and Innovation to Market Deployment," River Publishers Series in Communications, pp. 1-373, Jun 2014. 
[27] M. Pennacchioni, M. Di Benedette, T. Pecorella, C. Carlini, and P. Obino., "NB-IoT system deployment for smart metering: Evaluation of coverage and capacity performances," 2017 AEIT International Annual Conference, 2017, pp. 1-6, doi: 10.23919/AEIT.2017.8240561.

[28] J. Abdujalilov, K. Turzhanova, S. Konshin, A. Solochshenko, and B. Yakubov, "Analysis and improvement of the methods used for performance assessing of $4 \mathrm{G}$ network with NB-IoT technology for three scenarios of spectrum use in the $900 \mathrm{MHz}$ range," 2020 International Conference on Information Science and Communications Technologies (ICISCT), 2020, pp. 1-4.

[29] I. B. Oluwafemi and O. J. Femi-Jemilohun, "Suburban Area Path Loss Propagation Prediction and Optimization at 900 and 1800 MHz," Journal of Engineering and Applied Sciences, vol. 13, pp. 2521-2529, 2018.

[30] A. Zreikat and M. Dordevic, "Performance Analysis of Path Loss Prediction Models in Wireless Mobile Networks in Different Propagation Environments," in The 3rd World Congress on Electrical Engineering and Computer Systems and Science, Jun 2017.

[31] O. Liberg, M. Sundberg, Y. P. E. Wang, J. Bergman, and J. Sachs, "Cellular Internet of Things: Technologies, Standards, and Performance," Elsevier Inc.: Amsterdam, The Netherlands, pp. 345-347, 2017.

\section{BIOGRAPHIES OF AUTHORS}
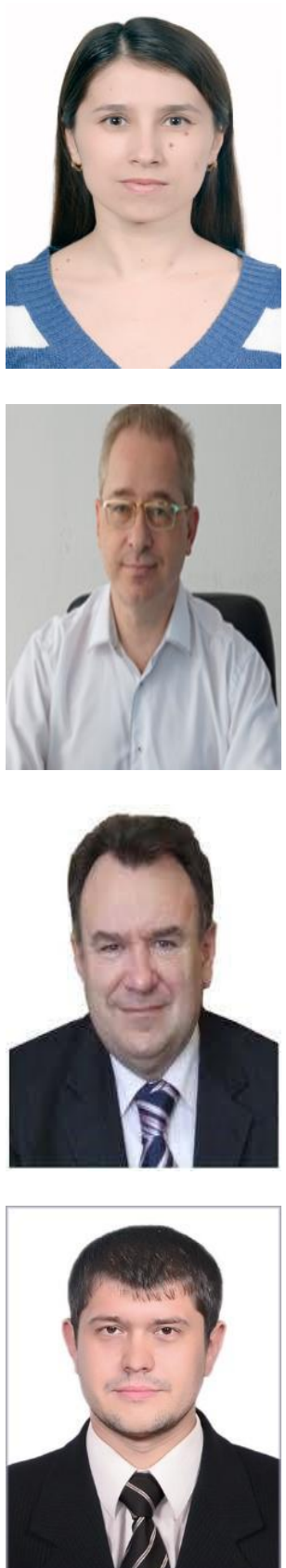

Karina Turzhanova is a PhD student, Master of telecommunication in Almaty University of Power Engineering and Telecommunications named after Gumarbek Daukeev, Kazakhstan. Her current areas of professional experience and interests include: NB-IoT technology, $5 \mathrm{G}$ networks, and video surveillance.

Sergey Konshin is a Lecturer, PhD. of technical science, Professor of Almaty University of Power Engineering and Telecommunications (AUPET, Kazakhstan). He has written more than 120 academic and research papers.

Valery Tikhvinskiy received the $\mathrm{PhD}$ degree in Radio engineering in 1988 and Doctor of Economics Science in 2003. He is a professor at the Moscow Technical University of Communications and Informatics and the Plekhanov University of Economics (2013). He also joined the International Information Technologies University (Kazakhstan) as a visiting professor. He is the author and co-author of at least 230 scientific papers and 11 books.

Alexandr Solochshenko is a $\mathrm{PhD}$ student. He received a Master's degree in Telecommunications from the Almaty University of Power Engineering and Telecommunications named after Gumarbek Daukeev (AUPET, Almaty, Kazakhstan) in 2017. His scientific interests include telecommunications, self-organizing networks, and fractal antennas. 